\title{
WEAK AND STRONG VIBRATION LOCALIZATION IN DISORDERED STRUCTURES: A STATISTICAL INVESTIGATION
}

\author{
C. Pierre \\ Department of Mechanical Engineering and Applied Mechanics, The University of Michigan, \\ Ann Arbor, Michigan 48109-2125, U.S.A.
}

(Received 26 July 1988, and in revised form 17 August 1989)

\begin{abstract}
A statistical investigation of the effects of disorder on the dynamics of one-dimensional nearly periodic structures is presented. The problem of vibration propagation from a local source of excitation is considered. While for the ordered infinite system there exists a frequency passband for which the vibration propagates without attenuation, the introduction of disorder results in an exponential decay of the amplitude for all excitation frequencies. Analytical expressions for the localization factors (the exponential decay constants) are obtained in the two limiting cases of weak and strong internal coupling, and the degree of localization is shown to depend upon the disorder to coupling ratio and the excitation frequency. Both modal and wave propagation descriptions are used. The perturbation results are verified by Monte Carlo simulations. The phenomena of weak and strong localization are evidenced. While the former affects little the dynamics of most engineering structures, the latter is shown to be of significant importance in structural dynamics.
\end{abstract}

\section{INTRODUCTION}

Mode localization in structures has received significant attention in the structural dynamics community lately (see references [1-11] or the review paper by Ibrahim [12]). This phenomenon occurs in repetitive structures, the periodicity of which is broken by small irregularities arising from unavoidable manufacturing and material tolerances. Under conditions of weak internal coupling for the structure of interest, such small disorder has drastic effects on its dynamical properties, by localizing the vibration modes to a small geometric region of the structure and confining the vibrational energy close to the source of excitation. In an average sense the decay of the vibration amplitude is exponential, the decay constant being referred to as the localization factor.

Localization may occur in a number of engineering structures such as blade assemblies $[1,2]$, multi-span structures $[3,4]$, and some large space structures $[5,6]$. The consequences of localization can be either damaging -as localized vibrations lead to larger amplitudes--or beneficial, as a means of passive control of vibration propagation. For practical purposes, localization is similar to damping as it manifests itself by a spatial decay of the vibration amplitude along the structure, even though for localized vibrations the energy is confined near the source of excitation, while for damping it is dissipated as it propagates. Thus, to an experimentalist unaware of disorder effects, localization could be easily mistaken for, and result in an overestimation of, damping.

Research on localization in structural dynamics has been mostly limited to deterministic analyses of the spatially localized free modes of disordered one-dimensional structures $[2,3,6-8]$. This is because analytical and even numerical (Monte Carlo) statistical investigations are difficult to conduct for the mode shapes. However, a consistent statistical 
treatment of localization can be formulated by studying the transmission of forced vibration from a local source of excitation. This is motivated by the argument that at a given frequency the localized mode shapes feature the same (exponential) spatial decay as the forced vibration patterns do [13]. To the knowledge of the author, two probabilistic investigations of localization have been carried out to date in structural dynamics, both approaching the problem from a forced excitation viewpoint. First, Hodges and Woodhouse [4] applied the work of Herbert and Jones [14] to calculate, by statistical perturbation methods, localization factors for a (finite) stretched string with irregularly spaced masses attached to it. However, they did not perform a systematic study of the dependence of the localization factor on the excitation frequency or the system parameters. Their research, though, exhibited the occurrence of two types of localization-weak and strongdepending on the magnitude of internal coupling in the structure, but they did not elaborate. Second, in a recent study, Kissel $[10,11]$ chose a wave propagation description to calculate localization factors for several infinite one-dimensional structures. Modeling the structures by random transfer matrices, he followed the approach originally proposed by Matsuda and Ishii [13] and Ishii [15], who were first to apply Furstenberg's theorem on the limiting behavior of products of random matrices. He systematically studied the dependence of the localization factor inside the passbands on the system parameters and the wave frequency in the limiting case of weak disorder. However, the structural models he chose (such as the spring-mass chain) did not allow him to vary the relative magnitudes of the internal coupling and the disorder, thereby restricting his findings mostly to weak localization, which does not affect the dynamics of most engineering structures significantly.

In addition, we must mention the detailed study of the resistance of one-dimensional chains of atoms by Kirkman and Pendry [16]. For a mathematical model nearly equivalent to ours, they derived expressions for the localization factor in the cases of weak and strong disorder through the use of a wave formulation. We also recommend the following works to the reader interested in localization: the comprehensive study of continuously disordered one-dimensional systems by Scott [17] and the in-depth review of localization and its relationship to diffusive transport theories such as statistical energy analysis by Hodges and Woodhouse [18].

This paper is concerned with the localization phenomenon for a generic model of one-dimensional nominally periodic structures; namely, the undamped chain of singleDOF oscillators shown in Figure 1. This system is chosen because, contrary to a spring-
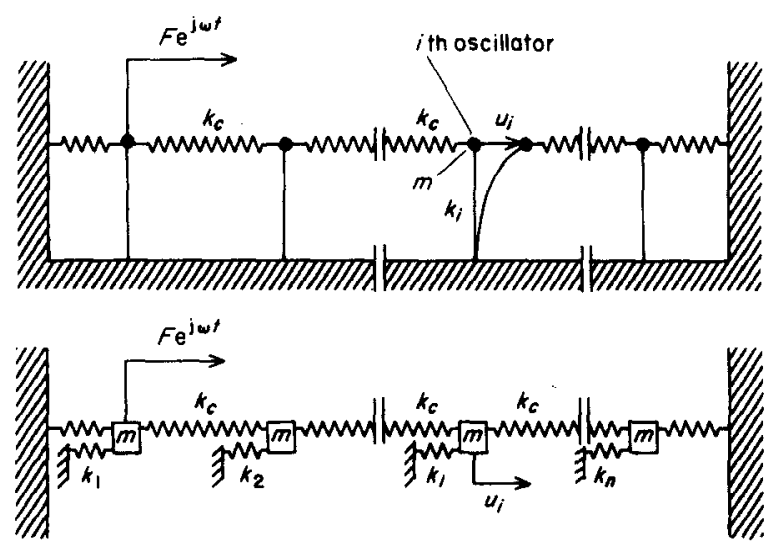

Figure 1. Two equivalent representations of an assembly of coupled oscillators. 
mass model, it allows one to vary the relative orders of magnitude of coupling and disorder, and thus it exhibits both weak and strong localization. To highlight the effects of disorder, we do not include damping in the model (although we recognize that all structures are damped and the uncertainty in damping is often large). A statistical treatment of the structural irregularities is chosen.

In the first part of the paper the techniques used to calculate the localization factors for a structure under forced excitation are reviewed. In the second part, the ordered structure is studied, and the concepts of frequency passband and stopband are illustrated by calculating the corresponding exponential amplitude decays. Statistical perturbation methods are utilized in the third part of the paper to derive analytical expressions for the localization factors of disordered systems in the two limiting cases of weak and strong coupling. In the fourth part, analytical results are compared to Monte Carlo simulations, and the validity of the two asymptotic results is discussed. The contributions of the present study are threefold. First, results of general significance to the theory of localization in engineering structures are presented. Although some of these results have appeared previously in the solid state physics literature, they are derived here in a structural dynamics context, and the practical implications for structures are strongly emphasized. The second contribution is the in-depth examination of the frequency dependence of localization effects in the weak and strong regimes. Finally, the distinction between weak and strong localization effects, which often seems to be confused in the literature on localization in structures, is clarified. In particular, the importance of strong localization in engineering structures is evidenced relative to that of weak localization.

\section{METHODOLOGIES}

\subsection{EQUATIONS OF MOTION}

The undamped assembly of $N$, coupled, single-DOF oscillators shown in Figure 1 is considered. The corresponding cyclic assembly has been used as a one-component mode representation of a continuously shrouded blade assembly [2]. Fixed-fixed boundary conditions are considered, even though the results are believed to hold for any nondissipative end condition. The system is excited at its left end by a simple harmonic force of frequency, $\omega$, and the transmission of steady state vibrations along the assembly is of interest. The equations of harmonic motion are

$$
-\omega^{2} m u_{i}=-k_{c}\left(2 u_{i}-u_{i-1}-u_{i+1}\right)-k_{i} u_{i}+F \delta_{i}^{1}, \quad i=1, \ldots, N,
$$

where $u_{i}$ is the displacement amplitude of the $i$ th oscillator, with $u_{0} \equiv u_{N+1} \equiv 0, k_{i}$ the $i$ th oscillator's stiffness, $k_{c}$ the coupling stiffness, $F$ the harmonic force amplitude, and $\delta_{i}^{\prime}$ the Kronecker symbol. For a periodic chain, $k_{i} \equiv k$, where $k$ is the nominal oscillator's stiffness. For the disordered structure the $k_{i}$ s are taken to be identically and uniformly distributed independent random variables with mean $k$. A uniform disorder distribution is chosen primarily because it leads to simpler calculations, even though the methods of analysis and some of the results of the paper hold for any type of distribution.

Only disorder in the stiffnesses of the oscillators is considered, even though a fully disordered system would have random masses and coupling stiffnesses as well. However, the effects of random oscillators' masses and stiffnesses are believed to be essentially the same, as both affect the oscillators' natural frequencies. Also, random coupling stiffnesses, since they do not affect the oscillators' frequencies, lead only to the weak localization studied by Kissel [11]. Therefore, restricting the randomness to oscillators' stiffnesses captures all the important localization effects in a relatively simple analysis. 
The equations of motion are made dimensionless by introducing $\omega_{h}=\sqrt{k / m}$, the nominal oscillator's frequency, $\bar{\omega}=\omega / \omega_{b}$, the dimensionless excitation frequency, $\Delta f_{i}=$ $\left(k_{i}-k\right) / k$, the dimensionless disorder for the $i$ th oscillator, and $R=k_{c} / k$, the square of the ratio of the coupling frequency to the nominal oscillator's frequency, or the dimensionless coupling, yielding

$$
\left(-\bar{\omega}^{2}[I]+[A]\right)\left(\begin{array}{c}
u_{1} \\
u_{2} \\
\vdots \\
u_{N}
\end{array}\right)=\left(\begin{array}{c}
\bar{F} \\
0 \\
\vdots \\
0
\end{array}\right),
$$

where $\bar{F}=F / k,[I]$ is the identity matrix, and $[A]$ is an $N \times N$ tridiagonal matrix given by

$$
[A]=\left(\begin{array}{ccccc}
\ddots & \ddots & 0 & \cdots & 0 \\
\ddots & \ddots & \ddots & 0 & \vdots \\
0 & -R & 1+\Delta f_{i}+2 R & -R & 0 \\
\vdots & 0 & \ddots & \ddots & \ddots \\
0 & \cdots & 0 & \ddots & \ddots
\end{array}\right)
$$

\subsection{MODAL FORMULATION}

To study vibration propagation, the amplitude at the right end is calculated when the system is driven at the left end. The approach has been originally proposed by Herbert and Jones [14] and then used by Hodges and Woodhouse [4]. From equation (2), the response amplitude of the $N$ th oscillator is

$$
u_{N} / \bar{F}=\left(-\bar{\omega}^{2}[I]+[A]\right)_{(N, 1)}^{-1}=[\text { cofactor matrix }]_{(1, N)} / \operatorname{det}\left(-\bar{\omega}^{2}[I]+[A]\right) .
$$

which holds only if $\bar{\omega}$ is not a natural frequency of the assembly. Remarkably, it follows from the tridiagonality of $[A]$ that the corresponding minor determinant is simply $(-R)^{N-1}$, and thus

$$
u_{N} / \bar{F}=R^{N-1} / \operatorname{det}\left(-\bar{\omega}^{2}[I]+[A]\right) .
$$

The above determinant is evaluated by modal analysis. The natural modes of the (ordered or disordered) system are determined by the eigenvalue problem

$$
\left(-\bar{\omega}_{r}^{2}[I]+[A]\right) \underline{u}_{r}=0, \quad r=1, \ldots, N,
$$

where $\bar{\omega}_{r}$ and $\underline{u}_{r}$ are the $r$ th frequency and eigenvector. The modal matrix [ $\left.U\right]$, the columns of which are the orthonormal eigenvectors, verifies $[U]^{\mathrm{T}}[U]=[I]$ and $[U]^{\mathrm{T}}[A][U]=\left[\operatorname{diag}\left(\bar{\omega}_{r}^{2}\right)\right]$, where $\mathrm{T}$ denotes a transpose. This yields

$$
\operatorname{det}\left(-\bar{\omega}^{2}[I]+[A]\right)=\operatorname{det}\left([U]^{\top}\left(-\bar{\omega}^{2}[I]+[A]\right)[U]\right)=\prod_{r=1}^{N}\left(\bar{\omega}_{r}^{2}-\bar{\omega}^{2}\right)
$$

The expression for the (spatial) exponential decay of the amplitude is obtained by writing the modulus of the $N$ th amplitude as $\left|u_{N} / \bar{F}\right|=\exp \left(-\gamma_{N} N\right)$, where $\gamma_{N}$ is the rate of decay for a given chain of length $N$. This yields, upon combining equations (4) and (6),

$$
\gamma_{N}=-\frac{1}{N} \ln \left|\frac{u_{N}}{\bar{F}}\right|=-\frac{N-1}{N} \ln R+\frac{1}{N} \sum_{r=1}^{N} \ln \left|\bar{\omega}_{r}^{2}-\bar{\omega}^{2}\right| .
$$

This expression is exact for a finite chain with boundary conditions and does not necessarily imply that there is an actual exponential decay of the amplitude. Of interest 
in the case of exponential decay is the limiting behavior of $\gamma_{N}$ as the length of the structure, $N$, goes to infinity. If $\gamma_{N}$ admits a limit $\gamma=\lim _{N \rightarrow \infty} \gamma_{N}$, then $\left|u_{N} / \bar{F}\right| \sim \exp (-\gamma N)$ for $N$ large. The vibration amplitude decays exponentially with $N$ at the rate $\gamma$, referred to as the exponential decay constant (the real part of the propagation constant [19]), and the amplitude is governed by an exponential envelope. + From equation (7), one has

$$
\gamma=-\ln R+\lim _{N \rightarrow \infty} \frac{1}{N} \sum_{r=1}^{N} \ln \left|\bar{\omega}_{r}^{2}-\bar{\omega}^{2}\right|,
$$

an expression valid for both ordered and disordered systems. For disordered chains $\gamma$ is not obtained by taking an average over several realizations, but by letting the size of the system become infinite. In the limiting cases for which analytical expressions of $\gamma$ will be obtained, it will be shown that taking the limit of $\gamma_{N}$ as $N \rightarrow \infty$ is equivalent to averaging $\gamma_{N}$ over all disordered systems, indicating that $\gamma_{N}$ is a self-averaging random variable [11].

\subsection{WAVE PROPAGATION FORMULATION}

Exponential decay constants can also be calculated by a traveling wave formulation. The system of Figure 1 is considered, but no boundary conditions are prescribed, which is equivalent to assuming that the assembly is infinite. The $i$ th site consists of the $i$ th oscillator and of the coupling spring at its right (see Figure 2), while the state vector at the $i$ th site is defined by $\left[u_{i}, u_{i-1}\right]^{\mathrm{T}}$. A two by two displacement transfer matrix is defined that relates the states at adjacent sites

$$
\left[\begin{array}{c}
u_{i+1} \\
u_{i}
\end{array}\right]=\left[T_{i}\right]\left[\begin{array}{c}
u_{i} \\
u_{i-1}
\end{array}\right], \quad \text { where }\left[T_{i}\right]=\left[\begin{array}{cc}
\frac{1+\Delta f_{i}+2 R-\bar{\omega}^{2}}{R} & -1 \\
1 & 0
\end{array}\right] \text {. }
$$

Thus the state at site $N+1$ is related to that at site 1 by

$$
\left[\begin{array}{c}
u_{N+1} \\
u_{N}
\end{array}\right]=\prod_{i=N}^{1}\left[T_{i}\right]\left[\begin{array}{l}
u_{1} \\
u_{0}
\end{array}\right]
$$

For an ordered chain all transfer matrices are identical, given by

$$
\left[T_{0}\right]=\left[\begin{array}{cc}
\frac{1+2 R-\bar{\omega}^{2}}{R} & -1 \\
1 & 0
\end{array}\right]
$$

For a disordered system the $\left[T_{i}\right] \mathrm{s}$ are random transfer matrices, and $\left[T_{0}\right]$, the transfer matrix for the ordered system, is their average matrix.

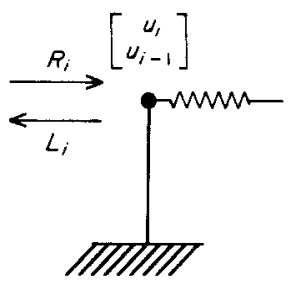

Site $i$

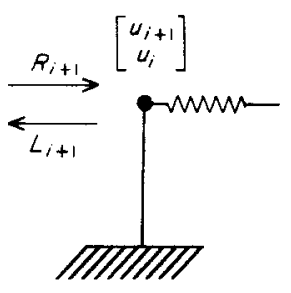

Site $i+1$

Figure 2. Site representation for wave formulation.

\footnotetext{
+ We note that damping, similarly to disorder, would result in a spatial exponential decay of the amplitude. However, the rate of decay $\gamma_{N}$ in equation (7) is solely due to disorder. The combined effects of disorder and damping will be the subject of future papers.
} 
Next wave amplitudes are introduced by a change of coordinates. Waves of frequency $\bar{\omega}$ are considered, the amplitudes of left and right traveling waves being denoted by $L$ and $R$, respectively. The complex amplitudes of the waves entering (resp. leaving) the site $i$ are $R_{i}$ and $L_{i+1}$ (resp. $L_{i}$ and $R_{i+1}$ ), as shown in Figure 2. The oscillators' displacement amplitudes are related to the wave amplitudes by $[10,15,19,20]$

$$
\left[\begin{array}{c}
u_{i} \\
u_{i-1}
\end{array}\right]=[P]\left[\begin{array}{c}
L_{i} \\
R_{i}
\end{array}\right] \quad \text { with }[P]=\left[\begin{array}{cc}
1 & 1 \\
\mathrm{e}^{-\mathrm{jk} k} & \mathrm{e}^{\mathrm{j} k}
\end{array}\right],
$$

where $j^{2}=-1$. The first of relations (12) states that the displacement of the $i$ th oscillator is the sum of the left and right traveling wave amplitudes. The complex $k$ is the wavenumber, a function of frequency $\bar{\omega}$. As shown in the next section, $k$ real defines a frequency passband, while $k$ of the form $\mathrm{j} \alpha$ or $\mathrm{j} \alpha+\pi$, where $\alpha$ is real, defines a stopband. Equations (9) and (12) lead to the introduction of a (mono-coupled) random wave transfer matrix that relates the wave amplitudes at sites $i$ and $i+1$ :

$$
\left[\begin{array}{l}
L_{i+1} \\
R_{i+1}
\end{array}\right]=\left[W_{i}\right]\left[\begin{array}{c}
L_{i} \\
R_{i}
\end{array}\right], \quad \text { where }\left[W_{i}\right]=[P]^{-1}\left[T_{i}\right][P]
$$

Note that $[P]^{-1}$ is not defined for $k=0$ and $\pi$ (corresponding to the passband edges). It is shown in the next section that the columns of the transformation matrix $[P]$ are the eigenvectors of the transfer matrix for the ordered chain, $\left[T_{0}\right]$. Hence for a periodic system $\left[W_{i}\right] \equiv\left[W_{0}\right]$, a diagonal matrix made of the eigenvalues of $\left[T_{0}\right]$. A general expression for $\left[W_{i}\right]$ can be obtained for frequencies in the passband ( $k$ real) by considering the waves entering and leaving a site in Figure 2:

$$
\left[W_{i}\right]=\left[\begin{array}{cc}
1 / t_{i} & -r_{i} / t_{i} \\
-r_{i}^{*} / t_{i}^{*} & 1 / t_{i}^{*}
\end{array}\right]
$$

Here ${ }^{*}$ denotes a complex conjugate, and $t_{i}$ and $r_{i}$ are the (complex) transmission and reflection coefficients for the $i$ th site, such that $\left|t_{i}\right|^{2}+\left|r_{i}\right|^{2}=1$, since for this undamped system energy is conserved as it propagates (or, equivalently, since $\operatorname{det}\left[T_{i}\right]=\operatorname{det}\left[W_{i}\right]=1$ ).

For a periodic site $\left[W_{0}\right]$ is diagonal, there is no reflection $(r=0)$, and the eigenvalues of $\left[T_{0}\right]$ are $t$ and $1 / t$. For frequencies in the passband $t$ has modulus one; thus there is no attenuation of the wave. For frequencies in the stopband (wavenumber not real), the transmission coefficient is real. Thus in a stopband of the ordered system there is no reflection but since the magnitude of the transmission coefficient is not one there is attenuation.

For a disordered site $\left[W_{i}\right]$ is not diagonal because $[P]$ is the eigenvector matrix for the average, not the disordered, site and cannot diagonalize $\left[T_{i}\right]$. Therefore there is reflection $\left(r_{i} \neq 0\right)$ in the disordered system. This multiple scattering over many sites results in localization.

The above formalism can be used to calculate the decay constant for an incident wave [15]. An infinite system is considered, such that the sites $i$ for $i=-\infty, \ldots, 0$ are ordered, the sites for $i=1, \ldots, N$ are disordered, and the ones for $i=N+1, \ldots, \infty$ are ordered. In other words, an $\mathrm{N}$-site disordered system embedded in an otherwise ordered infinite system is considered. A wave of amplitude $\alpha$ is incident from $-\infty$. For $i=-\infty, \ldots, 0$ and $i=N+1, \ldots, \infty$ there is no reflection because the wave transfer matrix is diagonal, and in a passband the wave travels unattenuated. For $i=1, \ldots, N$, though, multiple scatterings occur, and only part of the incident wave's energy is transmitted through the disordered segment. From equation (13), the wave amplitudes entering and leaving the disordered 
segment are related by, in a frequency passband,

$$
\left[\begin{array}{l}
L_{N+1} \\
R_{N+1}
\end{array}\right]=\left[\mathscr{W}_{N}\right]\left[\begin{array}{l}
L_{1} \\
R_{1}
\end{array}\right], \quad\left[\mathscr{W}_{N}\right]=\left[\begin{array}{cc}
1 / \tau_{N} & -\rho_{N} / \tau_{N} \\
-\rho_{N}^{*} / \tau_{N}^{*} & 1 / \tau_{N}^{*}
\end{array}\right]
$$

where $\left[\mathscr{W}_{N}\right]=\prod_{i=N}^{1}\left[\mathscr{W}_{i}\right]$, of determinant unity, is the wave transfer matrix for the $N$ disordered sites, and $\tau_{N}$ and $\rho_{N}$ the corresponding transmission and reflection coefficients. Since a wave of amplitude $\alpha$ is incident from the left and there is no reflection in the ordered segments, $L_{N+1}=0$ and $R_{1}=\alpha$. The ratio of transmitted to incident amplitude, $R_{N+1} / R_{1}$, is of interest, and can easily be shown to be equal to the transmission coefficient for the disordered segment, $\tau_{N}$. It follows that the rate of exponential decay of the transmitted wave amplitude is

$$
\gamma_{N}=-\frac{1}{N} \ln \left|\frac{R_{N+1}}{\alpha}\right|=-\frac{1}{N} \ln \left|\tau_{N}\right|
$$

and the limiting value of the exponential decay constant for the intinite disordered chain is

$$
\gamma=\lim _{N \rightarrow \infty}-\frac{1}{N} \ln \left|\tau_{N}\right|
$$

Thus, asymptotically, the ratio of transmitted to incident amplitude (or the modulus of the transmission coefficient) is governed by the envelope $\exp (-\gamma N)\left(\left|\tau_{N}\right| \sim \exp (-\gamma N)\right)$. The transmitted energy decays exponentially as well. Obviously, in the passband of an ordered system, $\gamma=0$ since $\rho_{N}=0$ and $\left|\tau_{N}\right|=1$; that is, there is pure energy transmission and no localization.

\section{A REVIEW OF THE ORDERED ASSEMBLY}

Both finite and infinite periodic chains have been investigated extensively by wave propagation techniques (see references [19-21] for example). Here the propagation constant $\gamma$ is computed for an infinite chain over the entire frequency spectrum by the modal approach as well as by the wave method. Equivalence of the results is proven.

\subsection{MODAL APPROACH}

The decay constant is given by equation (8), where $\bar{\omega}_{r}$ is the $r$ th frequency of the ordered chain with fixed-fixed ends, which can be shown to be [22]

$$
\bar{\omega}_{r}^{2}=1+2 R\left(1-\cos \frac{r \pi}{N+1}\right), \quad r=1, \ldots, N .
$$

Substituting equation (18) into equation (8) yields, with the limit of the series transformed into an integral,

$$
\gamma=-\ln R+\frac{1}{\pi} \int_{0}^{\pi} \ln \left|1+2 R(1-\cos x)-\bar{\omega}^{2}\right| \mathrm{d} x .
$$

The spectrum becomes continuous as $N \rightarrow \infty$, the frequencies of the infinite chain lying densely in the band $] 1,1+4 R$ [. Several cases are distinguished.

$0 \leqslant \bar{\omega}^{2}<1$ (stopband). One can show [23]

$$
\int_{0}^{\pi} \ln (\alpha+\beta \cos x) \mathrm{d} x=\pi \ln \frac{\alpha+\sqrt{\alpha^{2}-\beta^{2}}}{2}, \quad \alpha>|\beta| .
$$


Here $\alpha=1+2 R-\bar{\omega}^{2}$ and $\beta=-2 R$. After some manipulations, one obtains

$$
\gamma=\cosh ^{-1} \frac{1+2 R-\bar{\omega}^{2}}{2 R}, \quad 0 \leqslant \bar{\omega}^{2}<1,
$$

a strictly positive value corresponding to an attenuation of the amplitude for the infinite ordered chain. Accordingly, the frequency range is referred to as a stopband. Note that $\gamma \rightarrow 0$ as $\bar{\omega}^{2} \rightarrow 1$.

$\bar{\omega}^{2}>1+4 R$ (stopband). The expression (20) can still be used but with $\alpha=\bar{\omega}^{2}-1-2 R$ and $\beta=2 R$, yielding

$$
\gamma=2 \cosh ^{-1} \sqrt{\frac{\bar{\omega}^{2}-1}{4 R}}, \quad \bar{\omega}^{2}>1+4 R,
$$

a positive quantity corresponding to a stopband as well. Also, $\gamma \rightarrow 0$ as $\bar{\omega}^{2} \rightarrow 1+4 R$.

$1 \leqslant \bar{\omega}^{2} \leqslant 1+4 R$ (passband). The integral cannot be evaluated as in expression (20), since then $|\alpha| \leqslant|\beta|$. The integral needed is [23]

$$
\int_{0}^{\pi} \ln |\cos \theta-\cos x| \mathrm{d} x=-\pi \ln 2,
$$

where $\cos \theta=\left(1+2 R-\bar{\omega}^{2}\right) / 2 R$, less or equal to one in absolute value. Even though there is a singularity at $x=\theta$ (that is, when the excitation frequency coincides with one of the natural frequencies of the infinite system), the integral converges. Substituting equation (23) into equation (19) yields

$$
\gamma=0, \quad 1 \leqslant \bar{\omega}^{2} \leqslant 4 R .
$$

This frequency range is consequently referred to as a passband, since for the infinite chain the vibration propagates without attenuation.

\subsection{WAVE APPROACH}

For the ordered chain the displacement transfer matrix is [ $\left.T_{0}\right]$ given by equation (11), the eigenvalues of which are

$$
\lambda_{1,2}=\frac{1+2 R-\bar{\omega}^{2}}{2 R} \pm \frac{1}{2 R} \sqrt{\left(1-\bar{\omega}^{2}\right)\left(1+4 R-\bar{\omega}^{2}\right)}
$$

(such that $\lambda_{1} \lambda_{2}=1$ ) and the eigenvectors of which are the columns of

$$
[P]=\left[\begin{array}{cc}
1 & 1 \\
1 / \lambda_{1} & 1 / \lambda_{2}
\end{array}\right] \text {. }
$$

The wave transfer matrix, $\left[W_{0}\right]=[P]^{-1}\left[T_{0}\right][P]$, is diagonal of diagonal elements $\lambda_{1}$ and $\lambda_{2}$. Thus the transmission coefficient for $N$ ordered sites is simply $\tau_{N}=1 / \lambda_{1}^{N}$, and the corresponding exponential decay constant $\gamma=\ln \left|\lambda_{1}\right|$.

For $\bar{\omega}^{2}$ in the passband $[1,1+4 R]$, the eigenvalues of $\left[T_{0}\right]$ are complex and, upon letting $\bar{\omega}^{2}=1+4 R \sin ^{2} k$ in equation (25), become $\lambda_{1,2}=\exp ( \pm 2 j k)$, where $2 k$ is the real wavenumber. This immediately yields $\gamma=\ln \left|\mathrm{e}^{2 \mathrm{j} k}\right|=0$.

For $\bar{\omega}^{2} \geqslant 1+4 R$, letting $\bar{\omega}^{2}=1+4 R \cosh ^{2} k(k \geqslant 0)$ in equation (25) leads to $\lambda_{1,2}=-\mathrm{e}^{ \pm 2 k}$, corresponding to complex wavenumbers $\pi-2 \mathrm{j} k$. The positive decay constant is $\gamma=$ $\ln \left|-\mathrm{e}^{2 k}\right|=2 k$, where $k=\cosh ^{-1} \sqrt{\left(\bar{\omega}^{-2}-1\right) / 4 R}$, a result identical to that obtained by the modal approach in equation (22).

Finally, for $\bar{\omega}^{2} \leq 1$, one has $\lambda_{1}=\left[\left(1+2 R-\bar{\omega}^{2}\right) / 2 R\right]+\sqrt{\left(1-\bar{\omega}^{2}\right)\left(1+4 R-\bar{\omega}^{2}\right) / 2 R} \geqslant 1$, and $\lambda_{2}=1 / \lambda_{1}$. The decay constant is $\gamma=\ln \left|\lambda_{1}\right|$ which, after some algebraic manipulations, can be shown to be identical to the modal result (21). 


\subsection{REMARKS}

The concepts of stopband and passband are illustrated in Figure 3, which displays the decay constant $\gamma$ versus frequency for various values of coupling. The passband width decreases proportionally to coupling and larger values of $\gamma$ are obtained in the stopbands as $R$ decreases, because vibration propagation becomes more difficult as the coupling between oscillators decreases.

While the wave calculation leads to identical amplitude decays for all ordered sites, computing $\gamma$ by the modal method requires one to take the limit as the size of the system goes to infinity, because boundary conditions are considered. These boundary conditions, always present in engineering structures, may influence $\gamma_{N}$ significantly and, for finite structures, the values of $\gamma$ given by equations $(21,22,24)$ would not be obtained exactly. Numerical simulations of equation (7) revealed that for fixed-fixed ends and $\bar{\omega}$ in the passband (not coinciding with a natural frequency), values of $\gamma$ as large as $10^{-2}$ were obtained for a 50-DOF system. Recall that $\gamma$ is zero for an infinite system. Thus the infinite approximation of finite structures is good in the sense that the values of $y$ in the stopbands are much larger than those in the passband, and that the results for a 50-DOF system, if plotted in Figure 3, would superpose the infinite system results almost exactly for the present scale. This means that the results of Figure 3 are essentially applicable to large periodic engineering structures with many (say more than 50) sites, such as blade assemblies and some truss-type structures. The infinite approximation, however, is poor in the sense that, when weak localization occurs, the localization factor $y$ for the disordered
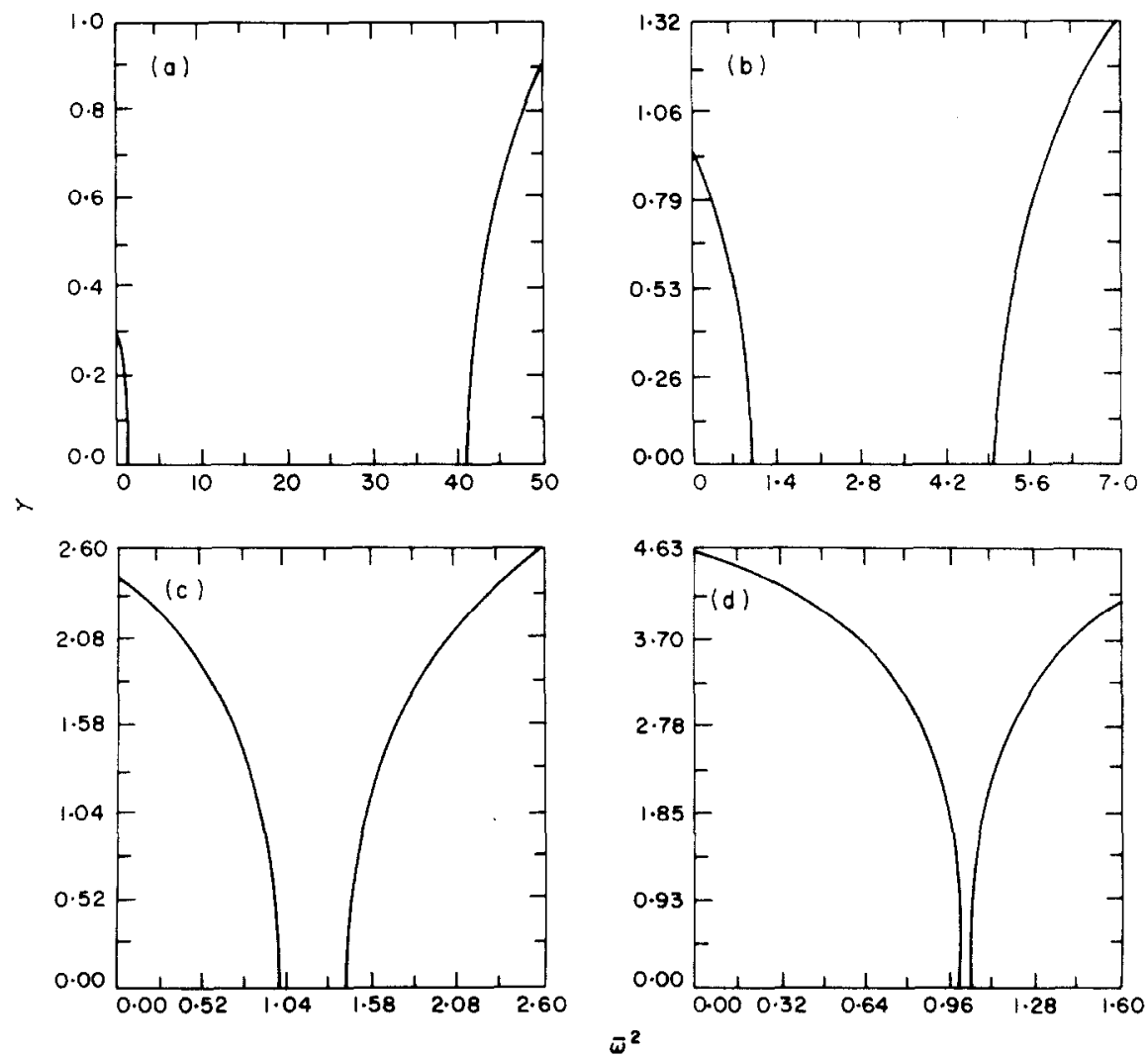

Figure 3. Exponential decay constant versus excitation frequency for ordered chains of oscillators with various internal couplings. (a) $R=10$; (b) $R=1$; (c) $R=0.1$; (d) $R=0.01$. 
infinite system is often less than $10^{-2}$. This makes such effects of disorder unimportant for many finite engineering structures, because boundary conditions alone result in values of $\gamma_{N}$ of comparable magnitude for ordered systems.

\section{A STUDY OF DISORDERED CHAINS BY STOCHASTIC PERTURBATION METHODS}

The decay constant $\gamma$ is calculated in terms of the excitation frequency when small disorder is present. Since localization phenomena will be shown to occur, $\gamma$ is referred to as the localization factor. The $\Delta f_{i}$ s are identically and uniformly distributed independent random variables with zero mean and standard deviation $\sigma=W / \sqrt{3}$, where $2 W$ is the width of the uniform probability density function.

In general, localization factors cannot be calculated in closed form, and one must resort to numerical Monte Carlo simulations to evaluate them. However, analytical results can be obtained in some limiting cases. This is achieved here through the use of perturbation methods. While these methods have been used to study the localization of the free modes $[3,5,8]$, here they are applied to the analysis of the forced response.

\subsection{SMALL Disorder to COUPLING RATIO-WEAK LOCALIZATION}

Here $O(\sigma / R)<1$, where $O(\cdot)$ denotes the order of the argument. Since disorder is small, it implies that the coupling is finite or large; hence this will be referred to as the strong coupling case. The wave approach is chosen here because it involves simpler calculations than the modal formulation, which requires the non-trivial expansion of the logarithm of a determinant [4]. The present development does not seem to have appeared in the literature for a chain of coupled oscillators. Kissel [10] has used the wave formulation for a sping-mass system (different from a chain of oscillators), but by an approximation to Furstenberg's theorem. A procedure that perturbs directly the wave transfer matrix is used here.

A classical perturbation scheme is applied to the wave formulation. The analysis is restricted to frequencies in the passband of the ordered system. The random displacement transfer matrix in equation (9) is written as

$$
\left[T_{i}\right]=\left[T_{0}\right]+\left[\Delta T_{i}\right] \quad \text { with } \quad\left[\Delta T_{i}\right]=\left[\begin{array}{cc}
\Delta f_{i} / R & 0 \\
0 & 0
\end{array}\right],
$$

where the unperturbed system is the ordered one and the perturbation is the disorder represented by $\left[\Delta T_{i}\right]$. For $1<\bar{\omega}^{2}<1+4 R$, the matrix of eigenvectors of $\left[T_{0}\right]$ is, from equation (26),

$$
[P]=\left[\begin{array}{cc}
1 & 1 \\
e^{-2 j k} & e^{2 j k}
\end{array}\right],
$$

where $k$ is defined by $\bar{\omega}^{2}=1+4 R \sin ^{2} k$, for $0<k<\pi / 2$. This leads to the random wave transfer matrix $\left[W_{i}\right]=[P]^{-1}\left[T_{i}\right][P]$, which can be written as

$$
\left[W_{i}\right]=\left[W_{0}\right]+\left[w_{i}\right]
$$

where, after calculations,

$$
\left[W_{0}\right]=\left[\begin{array}{cc}
\mathrm{e}^{2 \mathrm{j} k} & 0 \\
0 & \mathrm{e}^{-2 \mathrm{j} k}
\end{array}\right] \quad \text { and } \quad\left[w_{i}\right]=\frac{-\mathrm{j} \Delta f_{i}}{2 R \sin 2 k}\left[\begin{array}{cc}
\mathrm{e}^{2 \mathrm{j} k} & \mathrm{e}^{2 \mathrm{j} k} \\
-\mathrm{e}^{-2 \mathrm{j} k} & -\mathrm{e}^{-2 \mathrm{j} k}
\end{array}\right]
$$

are the unperturbed and random perturbation wave transfer matrices, respectively. Note that one must impose $k \neq 0, \pi / 2$ (that is, exclude the passband edges), since otherwise $\left[T_{0}\right]$ has a twofold eigenvalue and $[P]$ is singular. 
Next, the product of $N$ wave transfer matrices is taken and terms up to the first order in $\sigma / R$ are retained, yielding

$$
\left[W_{N}\right]=\prod_{i=N}^{1}\left(\left[W_{0}\right]+\left[w_{i}\right]\right) \simeq\left[W_{0}\right]^{N}+\sum_{i=1}^{N}\left[W_{0}\right]^{l-1}\left[w_{N-1+1}\right]\left[W_{0}\right]^{N-1}+O\left(\sigma^{2} / R^{2}\right)
$$

To obtain an approximation of the localization factor only the first diagonal element of $\left[\mathscr{W}_{N}\right], 1 / \tau_{N}$, is needed. One obtains, after some algebra,

$$
\frac{1}{\tau_{N}}=\mathrm{e}^{2 \mathrm{j} k N}-\frac{\mathrm{j} \mathrm{e}^{2 \mathrm{j} k N}}{2 R \sin 2 k} \sum_{i=1}^{N} \Delta f_{i}+O\left(\frac{\sigma^{2}}{R^{2}}\right) .
$$

By taking the modulus of $\tau_{N}, \gamma$ is obtained from equation (17) as

$$
\gamma=\lim _{N \rightarrow \infty} \frac{1}{2 N} \ln \left|1+\frac{1}{4 R^{2} \sin ^{2} 2 k} \sum_{l, m=1}^{N} \Delta f_{l} \Delta f_{m}+O\left(\frac{\sigma^{\top}}{R^{2}}\right)\right| .
$$

Note that the second order terms in equation (31) may lead to second order terms in the modulus as well, because this first order analysis does not capture all second order terms. However, it can readily be seen from equation (30) that these additional second order terms only involve products of the form $\Delta f_{l} \Delta f_{m}$ for $l \neq m$. Approximating $\ln (1+x)$ by $x$ in equation (32) leads to

$$
\gamma=\lim _{N \rightarrow \infty} \frac{1}{2 N}\left\{\frac{1}{4 R^{2} \sin ^{2} 2 k} \sum_{l, m=1}^{N} \Delta f_{l} \Delta f_{m}+O^{\prime}\left(\frac{\sigma^{2}}{R^{2}}\right)\right\},
$$

where the terms $O^{\prime}\left(\sigma^{2} / R^{2}\right)$ are of the form $\Delta f_{l} \Delta f_{m}$ for $l \neq m$. One has

$$
\lim _{N \rightarrow \infty} \frac{1}{N} \sum_{l, m=1}^{N} \Delta f_{l} \Delta f_{m}=\sigma^{2}
$$

because the $\Delta f_{i}$ s are uncorrelated and identically distributed. Hence all the terms $\Delta f_{i} \Delta f_{m}$ for $l \neq m$ do not contribute to the localization factor, justifying the fact that a first order perturbation analysis is sufficient. Combining equations (33) and (34) leads to

$$
\gamma=\frac{\sigma^{2}}{8 R^{2} \sin ^{2} 2 k}+O\left(\frac{\sigma^{4}}{R^{4}}\right), \quad 0<k<\frac{\pi}{2} .
$$

The localization factor is always greater than zero for disordered systems, while it is zero for the corresponding ordered system. Disorder results in an exponential attenuation of the wave amplitude as it propagates along the chain-the so-called localization, or confinement, phenomenon. Obviously, the degree of localization depends only on the disorder to coupling ratio and the excitation frequency. Note that these results are for undamped systems, thus the amplitude decay is created by disorder, not damping, resulting in a confinement, not a dissipation, of energy.

While $\gamma$ has been obtained by taking a limit as $N \rightarrow \infty$, it can also be calculated by taking the mathematical expectation of the logarithm of the transmission coefficient. Equation (31) gives

$$
\gamma=\left\langle\frac{1}{2 N} \ln \left|\frac{1}{\tau_{N}}\right|^{2}\right\rangle \simeq \frac{1}{2 N} \frac{1}{4 R^{2} \sin ^{2} 2 k} \sum_{l, m=1}^{N}\left\langle\Delta f_{l} \Delta f_{m}\right\rangle,
$$

which results in equation (35) because $\left\langle\Delta f_{l} \Delta f_{m}\right\rangle=\sigma^{2} \delta_{l}^{m}$, where $\langle\cdot\rangle$ denotes an average. Thus $\gamma$ can be regarded either as the localization factor for an infinite chain or as the average localization factor for finite (large) chains. Also, the assumption of uniform disorder has not been used and equation (35) is valid for other distributions as well. 
Next, the localization factor is written in terms of the excitation frequency by expressing the wave number $2 k$ as a function of $\bar{\omega}$. A little algebra gives

$$
\gamma^{(c)}=\sigma^{2} / 2\left(\bar{\omega}^{2}-1\right)\left(1+4 R-\bar{\omega}^{2}\right), \quad 1<\bar{\omega}^{2}<1+4 R,
$$

where the superscript $(c)$ stands for a classical perturbation result, which holds in the strong coupling case. The approximation $\gamma^{(c)}$ of $\gamma$ deteriorates as $\sigma / R$ increases, and eventually the classical perturbation method fails for finite perturbations $\sigma / R$ : that is, for weak coupling. Note that $\gamma^{(c)}$ also becomes large near, and goes to infinity at, the passband edges $\bar{\omega}^{2}=1$ and $1+4 R$. Thus one may expect the perturbation expression (37) to be a poor approximation of the localization factor near the stopbands.

The variation of $\gamma^{(c)}$ throughout the passband can be examined by introducing the parameter $\alpha$ defined by $\bar{\omega}^{2}=1+\alpha R, 0<\alpha<4$. Equation (37) can be rewritten as

$$
\gamma^{(c)}=\frac{\sigma^{2}}{R^{2}} \frac{1}{2 \alpha(4-\alpha)}, \quad 0<\alpha<4
$$

The variation of $\gamma^{(c)} /\left(\sigma^{2} / R^{2}\right)$ in terms of $\alpha$ is shown in Figure 4. The localization factor is minimum at midband $\left(\alpha=2\right.$, or $\left.\bar{\omega}^{2}=1+2 R\right)$ and becomes large near the stopbands. Equation (38) clearly shows that localization depends only on the ratio of disorder to coupling.

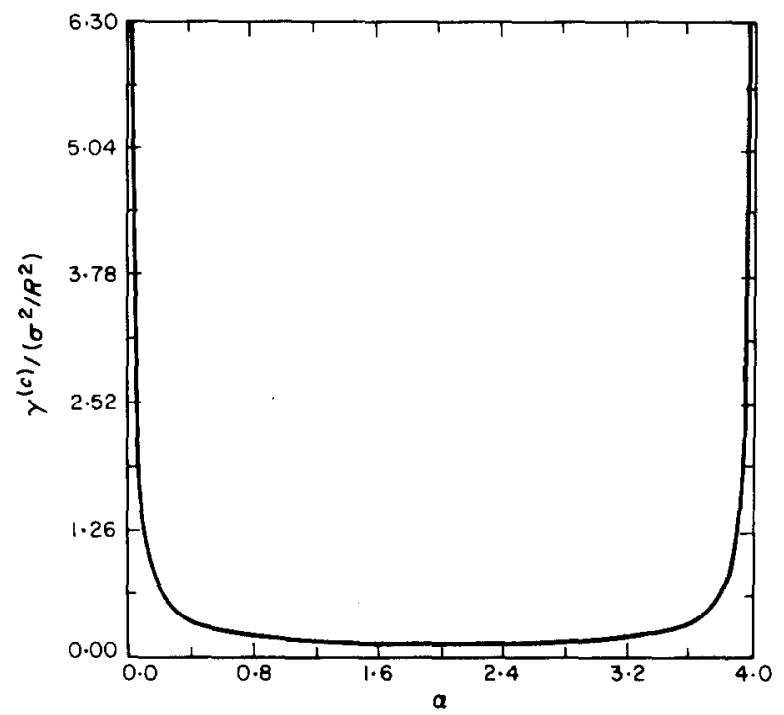

Figure 4. Variation of the localization factor in the frequency passband for a disordered system, by the classical perturbation method.

Localization factors could be computed as well for $\bar{\omega}$ in the stopbands. However, as will be shown by Monte Carlo simulations, disorder only slightly modifies the already large value of $\gamma$ in the stopbands, where strong attenuation occurs.

Finally, it is important to illustrate numerically the localization effect in the strong coupling case. For $R=1$ and $\sigma=10 \%$ (a large disorder), the localization factor at midband $\left(\bar{\omega}^{2}=3\right)$ is $\gamma^{(c)}=0.00125$. For an "infinite" system without boundary effects, the vibration amplitude is governed by $\mathrm{e}^{-\gamma^{(c)} N}$, and 555 sites are needed for the amplitude to decay by a factor two. Even though this effect can be regarded as significant because it simply results from disorder and is not caused by damping, it is likely to be rather unimportant for engineering structures that rarely comprise that many substructures and for which 
boundary and damping effects may be more significant. In real structures unintentional disorder rarely exceeds $3-4 \%$ and thus one may expect even smaller values of $\gamma$. This small effect of disorder in the strong coupling case is termed weak localization.

\subsection{IARGE DISORDER TO COUPLING RATIO-STRONG LOCALIZATION}

The other limiting case, $O(\sigma / R)>1$, is considered. Since disorder is always small, it implies that the coupling is weak. Then the classical perturbation method fails and one needs to introduce a modified scheme that treats the coupling as a perturbation, while disorder is included in the unperturbed system to avoid degeneracy. The unperturbed system then consists of an assembly of uncoupled, disordered oscillators. This modified scheme has been shown to analyze the localized free modes of several disordered structures effectively $[3,7,8]$, because strongly localized modes are essentially perturbations of decoupled modes. Here the method is applied to the forced vibration case. Note that the localization factor at midband has also been obtained by Hodges and Woodhouse [4] in the weak coupling case. Here it is calculated over the entire frequency spectrum.

\subsubsection{Modal approach}

The localization factor is calculated from equation (8), the natural frequencies being approximated by the following first order perturbation analysis. The system matrix $[A]$ is decomposed as $[A]=\left[A_{0}^{(m)}\right]+\left[a^{(m)}\right]$, where

$$
\left[A_{0}^{(m)}\right]=\left[\operatorname{diag}\left(1+\Delta f_{i}\right)\right] \quad \text { and } \quad\left[a^{(m)}\right]=\left(\begin{array}{ccccc}
\ddots & \ddots & 0 & \cdots & 0 \\
\ddots & \ddots & \ddots & 0 & \vdots \\
0 & -R & 2 R & -R & 0 \\
\vdots & 0 & \ddots & \ddots & \ddots \\
0 & \cdots & 0 & \ddots & \ddots
\end{array}\right)
$$

are the modified unperturbed and perturbation matrices. Perturbation theory for the eigenvalue problem [24] can be applied because the unperturbed eigenvalues are split by random disorder, and one can easily show [8] that the first order perturbed natural frequencies are $\bar{\omega}_{r}^{2(m)}=1+\Delta f_{r}+2 R$, for $r=1, \cdots, N$. This leads to the approximation of the localization factor

$$
\gamma^{(m)}=-\ln R+\lim _{N \rightarrow \infty} \frac{1}{N} \sum_{r=1}^{N} \ln \left|1+\Delta f_{r}+2 R-\bar{\omega}^{2}\right|,
$$

where $(m)$ refers to the modified perturbation scheme for weak coupling. Obviously the limit in equation (40) is the expected value of the logarithmic expression, and hence

$$
\gamma^{(m)}=-\ln R+\int_{-\infty}^{+\infty} p(\Delta f) \ln \left|1+2 R+\Delta f-\bar{\omega}^{2}\right| \mathrm{d}(\Delta f),
$$

where $p$ is the probability density function of the disorder. This is easiest to evaluate for a uniform distribution, even though it could be calculated numerically in other cases as well. One obtains

$$
\gamma^{(m)}=-\ln R+\frac{1}{2 W} \int_{-W}^{+W} \ln \left|1+2 R+\Delta f-\bar{\omega}^{2}\right| \mathrm{d}(\Delta f) .
$$

Equation (42) could also be obtained by considering the average decay $\gamma_{N}^{(m)}$ for finite assemblies:

$$
\left\langle\gamma_{N}^{(m)}\right\rangle=-\ln R+\frac{1}{N} \sum_{r=1}^{N}\left\langle\ln \left|1+\Delta f_{r}+2 R-\bar{\omega}^{2}\right|\right\rangle=\gamma^{(m)}
$$


Hence equation (42) can be regarded either as the localization factor for an infinite chain or as the average factor for finite systems. After integration, it yields

$$
\begin{aligned}
\gamma^{(m)}= & -\ln R-1+\frac{1+2 R+W-\bar{\omega}^{2}}{2 W} \ln \left|1+2 R+W-\bar{\omega}^{2}\right| \\
& -\frac{1+2 R-W-\bar{\omega}^{2}}{2 W} \ln \left|1+2 R-W-\bar{\omega}^{2}\right| .
\end{aligned}
$$

Since only first order coupling terms were retained in the natural frequencies, this expression is essentially a first order result in the coupling to disorder ratio, valid for frequencies in the passband and stopbands. At midband, $\bar{\omega}^{2}=1+2 R$, equation (44) reduces to

$$
\gamma_{\text {midband }}^{(m)}=\ln (\sigma / R)+\ln \sqrt{3}-1+O\left(R^{2} / \sigma^{2}\right)
$$

which shows that the localization factor depends only upon the coupling to disorder ratio. This approximation of $\gamma$, valid for $R / \sigma$ first order or smaller, shows that localization becomes more pronounced as the (small) coupling decreases and that $\gamma^{(m)} \rightarrow \infty$ as $R / \sigma \rightarrow 0$. This makes sense because $R / \sigma=0$ corresponds to the modified unperturbed system consisting of uncoupled oscillators, for which there is total attenuation from one site to another. A typical value is obtained for $R=0.01$ and $\sigma=3 \%$, given by $\gamma_{\text {midband }}^{(m)}=0.648$. This value is several orders of magnitude larger than the typical value obtained for the (weak) localization factor in the strong coupling case. A quick calculation reveals that, in an average sense, the vibration amplitude of the fourth oscillator is $\left|u_{4} / \bar{F}\right| \sim 0.075$ : that is, only $0.075^{2}=0.56 \%$ of the vibrational energy is transmitted to the fourth oscillator! For the ordered system the value of $\gamma$ at midband is zero and $100 \%$ of the energy is transmitted. This drastic phenomenon created by small disorder in the weak coupling case is referred to as strong localization.

\subsubsection{Wave approach}

This approximation of the localization factor can also be obtained through the use of the wave transfer matrix. Again the basic idea is to treat the coupling as a perturbation by writing $\left[T_{i}\right]=\left[T_{0 i}^{(m)}\right]+\left[\Delta T^{(m)}\right]$, where, from equation $(9) \dagger$,

$$
\left[T_{0 i}^{(m)}\right]=\left[\begin{array}{cc}
\frac{1+\Delta f_{i}+2 R-\bar{\omega}^{2}}{R} & 0 \\
0 & 0
\end{array}\right], \quad\left[\Delta T^{(m)}\right]=\left[\begin{array}{rr}
0 & -1 \\
1 & 0
\end{array}\right] .
$$

Since the unperturbed matrix is diagonal, no transformation is necessary to calculate the wave transfer matrix of the perturbed system, given by $\left[W_{i}^{(m)}\right]=\left[T_{i}\right]$. A first order approximation in the perturbation parameter, $R / \Delta f_{i}$, is obtained for the wave transfer matrix for $N$ sites:

$$
\left[\mathscr{W}_{N}\right]=\prod_{i-N}^{1}\left(\left[T_{0 i}^{(m)}\right]+\left[\Delta T^{(m)}\right]\right)=\prod_{i=1}^{N}\left[T_{0 i}^{(m)}\right]+\sum_{i-1}^{N}\left\{\left(\prod_{i=N}^{1+1}\left[T_{0 i}^{(m)}\right]\right)\left[\Delta T^{(m)}\right]\left(\prod_{i-1-1}^{1}\left[T_{0 i}^{(m)}\right]\right)\right\},
$$

† The term $2 R / R$ in the first diagonal element of the transfer matrix could also be considered to belong to the perturbation matrix instead of the unperturbed matrix. This would result in a slightly less accurate approximation of the localization factor. 
where the first diagonal element of that matrix is needed to calculate $\gamma$. A little algebra shows that the first order perturbation terms in equation (47) do not contribute to the first diagonal element, so that

$$
\left(\left[\mathcal{W}_{N}\right]\right)_{11} \simeq \prod_{i=1}^{N} \frac{1+\Delta f_{i}+2 R-\bar{\omega}^{2}}{R}
$$

and the approximation of the localization factor is, from equation (16),

$$
\gamma_{N}^{(m)}=\frac{1}{N} \sum_{i=1}^{N} \ln \left|\frac{1+\Delta f_{i}+2 R-\bar{\omega}^{2}}{R}\right|,
$$

which, upon taking the limit as $N \rightarrow \infty$ (or equivalently, taking the average for a finite chain), leads to

$$
\gamma^{(m)^{\prime}}=-\ln R+\frac{1}{2 W} \int_{-W}^{+u} \ln \left[1+x+2 R-\bar{\omega}^{2} \mid \mathrm{d} x=\gamma^{(m)},\right.
$$

which is identical to the approximate localization factor obtained by the modal approach (equations $(42,44))$.

\section{COMPARISON OF ANALYTICAL AND MONTE CARLO RESULTS}

This section's purpose is twofold: to analyze the dependence of the localization factor upon frequency, coupling and disorder, and to verify the validity of the analytical results by numerical simulations.

There are two choices for the Monte Carlo simulations. First, one can choose the modal formulation for a finite system of size $N$ with boundary conditions, and average the values of $\gamma_{N}$ obtained by equation (7) over a number of disordered systems, yielding the estimate $\gamma=\left\langle\gamma_{N}\right\rangle$. Second, the wave formulation can be adopted, the transmission coefficient $\tau_{N}$ being computed for a finite disordered segment but without boundary conditions by multiplying $N$ transfer matrices $\left[T_{i}\right]$ and applying the similarity transformation defined by $[P]$ to the resulting matrix. The estimate of the localization factor is then the average, over a number of realizations of the disordered segment, of $\gamma_{N}$ given by equation (16). The basic difference between the two techniques is that one accounts for boundary conditions, while the other does not. Because we are interested in engineering structures, the modal approach that simulates an actual finite structure with boundary conditions was chosen whenever possible, the limitations of this choice being discussed later. Another question that arises is how many realizations should be considered, and how large each system should be. These considerations are primarily governed by the computer cost. Monte Carlo simulations by the modal approach were found to be several orders of magnitude more expensive than those by the wave approach. This is because $r$ eigenvalue problems of size $N$ must be solved by the modal approach, where $r$ is the number of realizations, while the wave approach only demands $r$ times the multiplication of $N$ matrices. To keep the computer cost reasonable, 300 realizations of systems of size 60 were considered at most for a modal simulation. For the inexpensive wave simulation no such limitations were necessary, and two configurations were chosen: 1000 realizations for 300 disordered sites, or 6000 realizations for a single site. The rationale for choosing the simulation procedure is discussed below.

The variation of the localization factor in the passband, by classical perturbation method (equation (37)) and Monte Carlo simulation, for $R=1$ and $\sigma=1 \%$ is displayed in Figure 5 . Note the excellent agreement between the theoretical and numerical results over the entire passband. Also observe that $\gamma$ is largest near the stopbands, but that its magnitude 


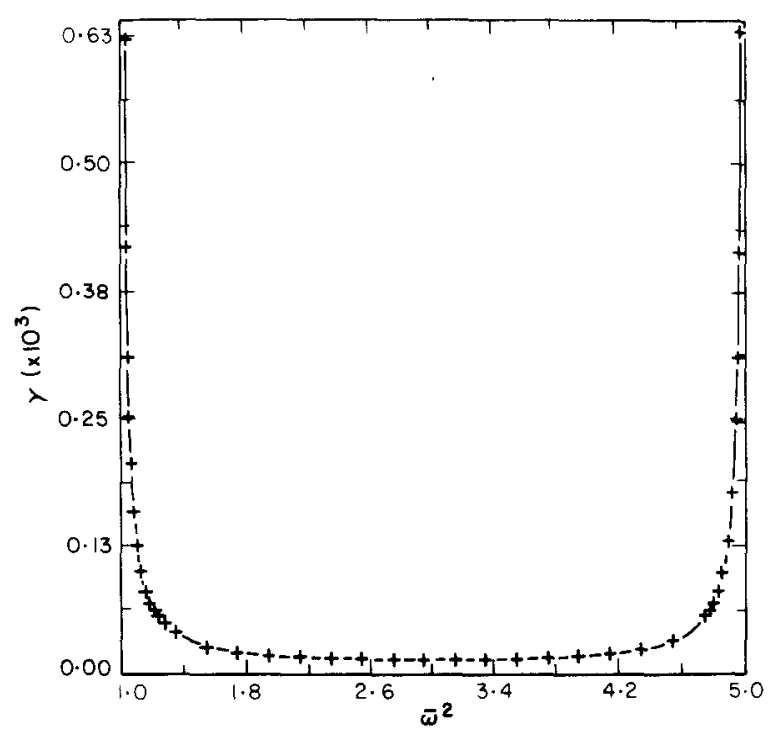

Figure 5. Localization factor in the frequency passband, for $\sigma=1 \%$ and $R=1$, by classical perturbation method (-) and Monte Carlo simulations (+).

is very small throughout the passband, indeed orders of magnitude smaller than $\gamma$ for the tuned system in the stopbands (which precludes us from displaying $\gamma$ in the stopbands).

Figures 6 and 7 are for a larger disorder to coupling ratio equal to $0 \cdot 1$. Figure 6 is for a $10 \%$ disorder and $R=1$, while Figure 7 is for a $1 \%$ disorder and $R=0 \cdot 1$. Almost identical values are obtained for $\gamma$, which is expected because the degree of localization depends only on the disorder to coupling ratio. From equation (37), $\gamma$ is 100 times greater than in Figure 5. The agreement between analytical and simulation results is also excellent, except near the stopbands where the perturbation result overpredicts the Monte Carlo result. This is explained by noting that the perturbation result becomes infinite at the

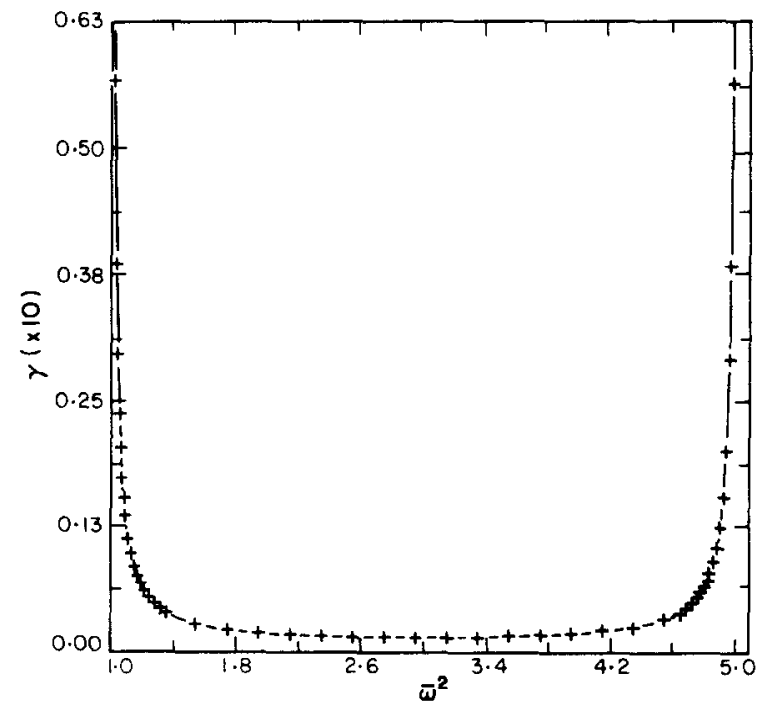

Figure 6. Localization factor for $\sigma=10 \%$ and $R=1$, by classical perturbation method ( - ) and Monte Carlo simulations $(+)$. 


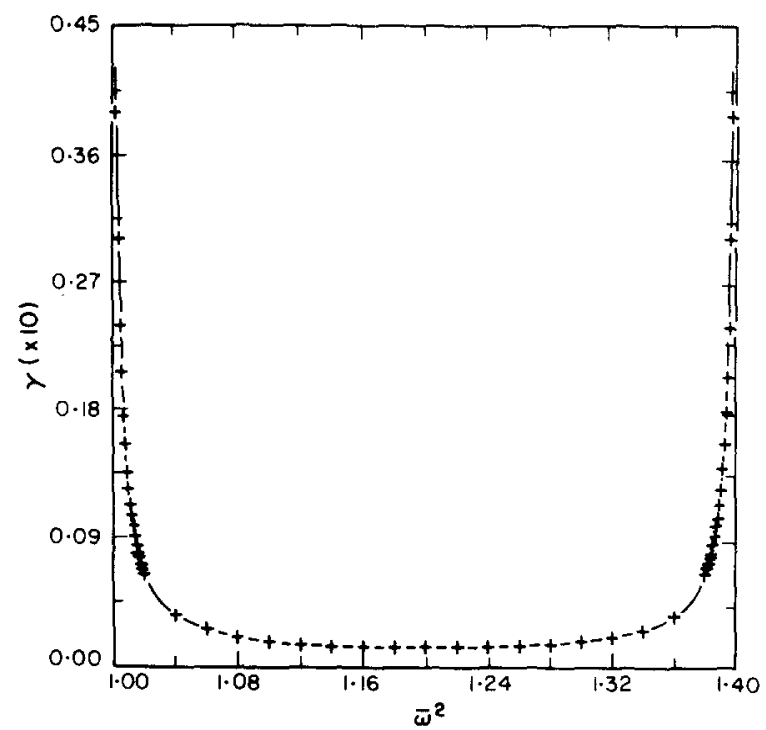

Figure 7. Localization factor for $\sigma=1 \%$ and $R=0 \cdot 1$, by classical perturbation method $(-)$ and Monte Carlo simulations $(+)$.

passband edges, and therefore this first order approximation deteriorates as the frequency approaches 1 or $1+4 R$. A second order approximation in the variance would lead to a better agreement near the stopbands. Also, as the ratio of disorder to coupling increases, the approximation of $\gamma$ becomes worse for a given frequency, while as the ratio decreases, the approximation becomes better closer to the edges of the passband. If $\bar{\omega}$ approaches an edge sufficiently, though, a discrepancy always occurs between the analytical and Monte Carlo result. Finally, it is shown in Figures 5-7 that for such small disorder to coupling ratios the effect of disorder is merely to extend the stopbands a little, as $\gamma$ inside the passband is quite small.

A wave formulation was chosen for the Monte Carlo simulations in Figures 5-7. Even though a modal calculation would have been preferred, it was found that the very small values of $\gamma$ inside the passband (mostly less than 0.004 ) could not be obtained. Cost considerations limited the size of the systems considered to 60 , and a rapid calculation shows that this leads to a (maximum) amplitude decay of $22 \%$ between the two ends. It was found, though, that boundary conditions influenced the value of $\gamma$ very significantly, and no meaningful result could be obtained for an ensemble of 300 realizations. The values of $\gamma$ obtained by the modal simulation ranged approximately from -0.005 to 0.005 , without any definite pattern, and the variance of the localization factor was much larger than the mean. This is not surprising when one recalls that for a 50-DOF ordered system $\gamma \mathrm{s}$ of magnitude 0.01 were obtained in the passband instead of zero! Therefore very small decays cannot be simulated with reasonable cost for finite structures. Note that this is not a failure of the modal approach to analyze weak localization. Rather, it means that the concept of weak localization is irrelevant for a number of finite structures, because boundary conditions influence the amplitude decay as much as, and sometimes more than, the disorder. Therefore, the results of Figures 5-7 are only for infinite structures (or at least such that $N$ is much larger than the localization scale $1 / \gamma$ ). Based on the experience acquired from Monte Carlo simulations, one can estimate that a localization factor of 0.004 would require a structure of at least 600 sites in order for the expected localization effect to take place in a given disordered structure. This remark shows that 
not only the average of the localization factor is of interest, but its variance as well, for one is interested in the behavior of individual disordered structures as much as in that of the average of an ensemble of structures. Localization effects are meaningful only if they occur for typical disordered systems, that is, only if the variance of $\gamma$ is much smaller than the mean. Finally, it was found that the modal approach could be used for 60-DOF systems only if $\gamma>0.08$. Otherwise the wave method had to be used.

A few more words are in order concerning the wave simulation results. As noted by Kissel [11], we found that taking the average of the logarithm of the transmission coefficient for one disordered site led to excellent results. It should be pointed out, though, that this is not valid when dealing with larger localization factors $(\gamma>0.05)$ and thus, in general, this simplified approach should not be a substitute for complete simulations performed by multiplying many transfer matrices.

The localization factor in terms of frequency for a disorder to coupling ratio of one is displayed in Figure 8. Here the larger values of $y$ allow us to display its variation in the stopband. One observes that the agreement between the classical perturbation result and the simulation is still fair in the middle in the passband, but deteriorates rapidly as $\bar{\omega}$ moves toward the edges of the passband, where the perturbation results overpredict $\gamma$ significantly. In the stopbands $\gamma$ is very close to the decay of the ordered chain, justifying the fact that perturbation expressions were obtained only in the passband. The Monte Carlo results were obtained by the modal approach for systems of size 60 , and very good agreement was observed with the wave simulation results performed on 1000 realizations of 300 matrices. Therefore, for structures made of 60 sites with $10 \%$ disorder and excited at midband, an average decay of $99.8 \%$ of the vibration amplitude from one end to the other can be expected.

In Figure 9 is shown the localization factor in a weak coupling case, for a disorder to coupling ratio equal to three. Note that the Monte Carlo results compare rather well with the modified perturbation results, considering that the disorder to coupling ratio is not large. As expected, the classical perturbation analysis gives very large values of $\gamma$ and yields erroneous results. Also, the localization factor for the disordered system varies

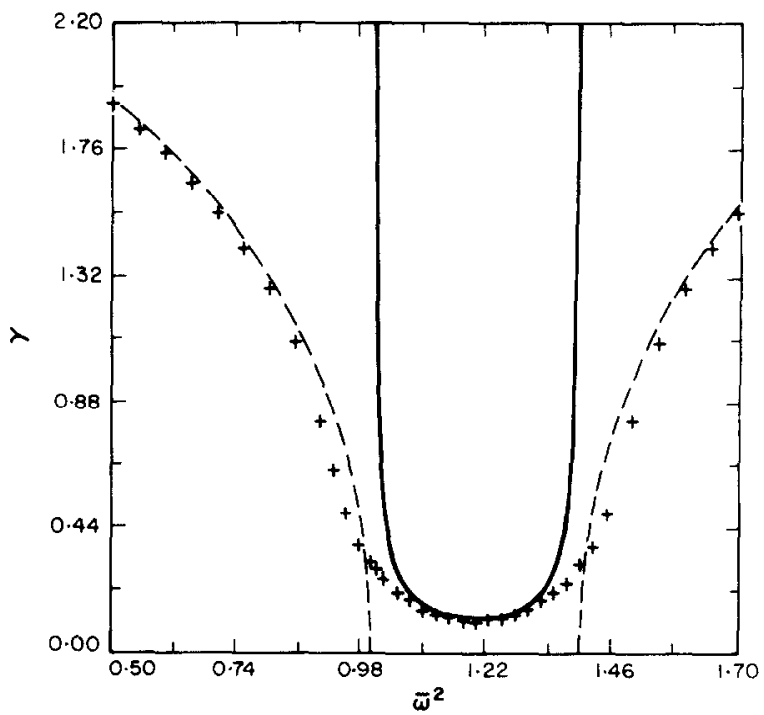

Figure 8. Localization factor in frequency passband and stopbands, for $\sigma=10 \%$ and $R=0 \cdot 1$, by classical perturbation method $(-)$ and Monte Carlo simulations $(+)$. The decay constant for the tuned system is also shown (- - ). 


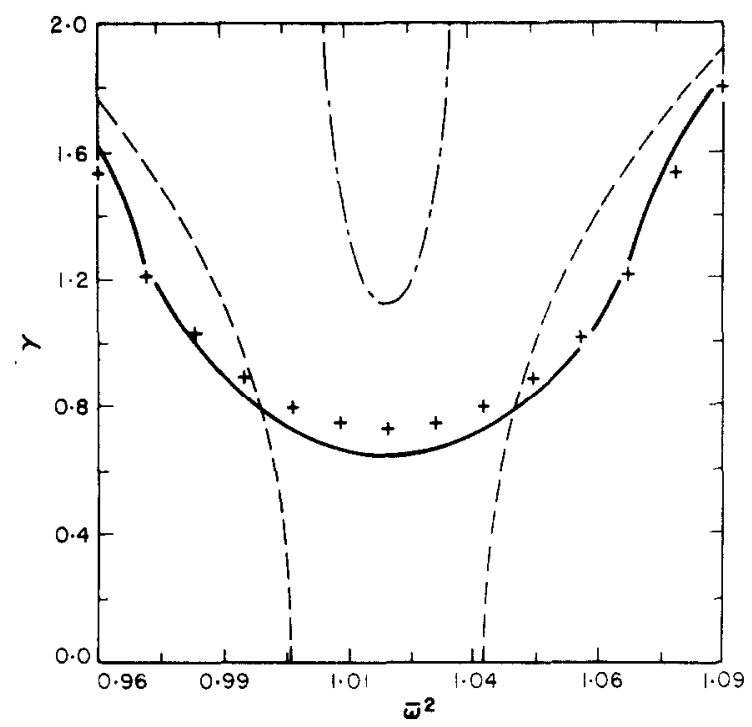

Figure 9. Localization factor in frequency passband and stopbands, for $\sigma=3 \%$ and $R=0 \cdot 01$, by modified perturbation method (-), Monte Carlo simulations (+), and classical perturbation method (- - - ). The decay constant for the tuned system is shown $(--)$.

little over the passband, and in the stopbands $\gamma$ is close to the tuned system's decay. Interestingly enough, in the stopband the decay for the disordered system is smaller than that for the tuned system, hence disorder decreases slightly the attenuation in the stopband! In the passband, however, the zero value of $\gamma$ for the tuned system becomes nearly 0.7 when small disorder is introduced, corresponding to a severe attenuation of the amplitude in this weak coupling case. It should be noted that the Monte Carlo results were obtained by simulating the modal formulation and agreed very well with the wave simulation results obtained by multiplying transfer matrices. The approach suggested by Kissel [11] to average over only one disordered site led to severe underestimates of $\gamma$, and it was concluded that it should not be used for large decays.

Figure 10 is for a larger disorder to coupling ratio equal to ten. Excellent agreement between the Monte Carlo and modified perturbation results (44) is observed. The classical perturbation results are so large that they could not be shown. If the plot were extended to include more of the stopbands it would show that the localization factor for tuned and mistuned systems approach each other as the distance to the passband increases. Note that the very large value of the localization factor, nearly equal to 1.9 , leads to a drastic decay over a few oscillators-the so-called strong localization. Also, $\gamma$ is almost constant over the frequency range shown, a very different behavior from that observed for weak localization, where the localization factor is largest in the vicinity of the stopbands. In fact, Figure 10 shows that the introduction of disorder practically eliminates the passband. Again, Monte Carlo results were obtained by simulating systems with boundary conditions.

Finally, in Figure 11 is shown a typical variation of the localization factor in terms of the disorder to coupling ratio, for a midband excitation. Monte Carlo simulation and classical and modified perturbation results are shown. As $\sigma / R$ increases from zero to approximately one, the simulation and classical perturbation results are in excellent agreement, corresponding to the phenomenon of weak localization. This agreement deteriorates as the disorder to coupling ratio further increases, and while the classical perturbation results diverge from the numerical solution, the latter approaches the 


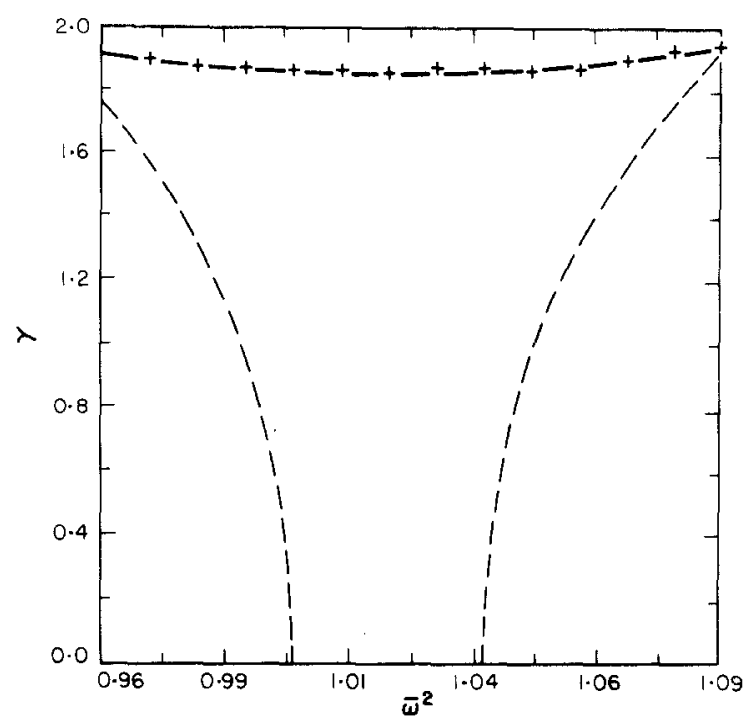

Figure 10. Localization factor for $\sigma=10 \%$ and $R=0.01$, by modified perturbation method ( - ) and Monte Carlo simulations $(+)$. The decay constant for the tuned system is shown (-- ).

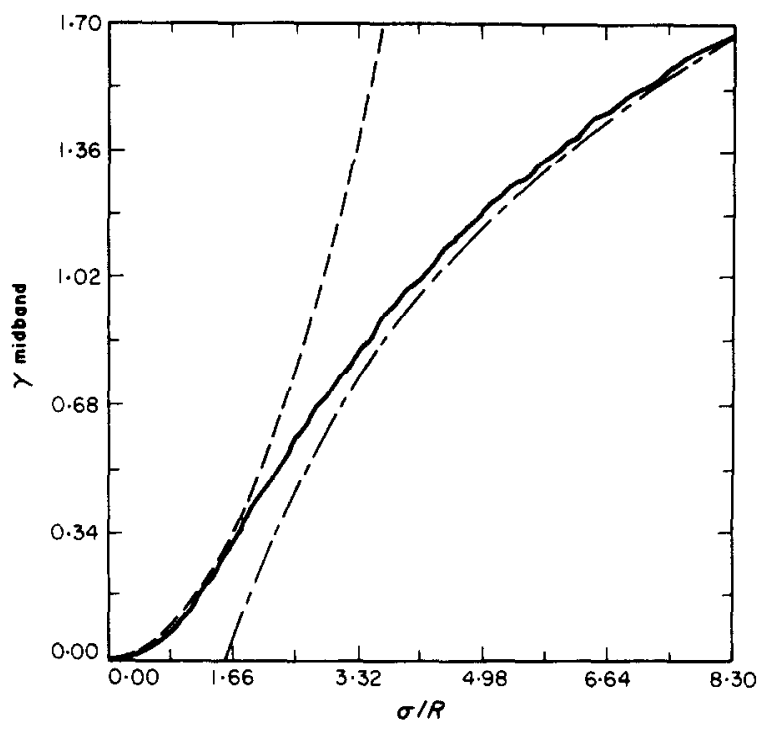

Figure 11. Localization factor versus disorder to coupling ratio, at midband frequency $\bar{\omega}^{2}=1+2 R$, by Monte Carlo simulations (-), classical perturbation method $(---)$, and modified perturbation method (- - $)$.

modified perturbation results. For $\sigma / R>6$ very good agreement is observed between the Monte Carlo and the modified perturbation results, corresponding to strong localization. The agreement would improve as the disorder to coupling ratio further increases. The region where neither perturbation scheme gives accurate results (approximately $1.5<$ $\sigma / R<3$ ) corresponds to the transition between weak and strong localization, for which significant effects of disorder begin to occur. These intermediate cases require a numerical simulation of the effects of disorder. Finally, note that the Monte Carlo results are a little jagged because of cost limitations in the simulations. Smoother results could be obtained, but the resulting additional expense was not deemed worthwhile. 


\section{ON WEAK AND STRONG LOCALIZATION}

Weak localization occurs in systems with strong (finite) internal coupling and weak disorder, for which the amplitude decay per site is very small. Even though of considerable academic interest, weak localization is probably of little concern to the structural dynamicist, based on the three following arguments. First, even for an "infinite" structure without boundaries, the decay per site is very small. For example, a localization factor of 0.005 corresponds to a localization length of 200 sites, and 140 substructures are needed for the wave amplitude to decay by a factor two. Typically, the localization effect is significant for chains consisting of several hundreds or thousand of sites, making it relevant to systems such as atom chains and lattices studied in physics. Second, Monte Carlo simulations have shown that boundary conditions can affect the very small values of the localization factors significantly, and in many cases obscure any noticeable effect of disorder. This happens when the size of the structure is not much larger than the localization length. Third, it is believed that the light damping that exists in all structures provides spatial amplitude decays that are at least comparable in magnitude to those created by disorder. This will be discussed in a future paper. In engineering, the typical number of component systems making up a periodic structure is much smaller than in solid state physics (say less than one hundred), therefore making weak localization of little interest. A notable exception, however, could be truss-type structures that may consist of many components.

Strong localization, on the other hand, occurs in weakly coupled, weakly disordered systems, and is a drastic phenomenon: for a strongly localized vibration only a few oscillators (say less than ten) participate in the motion of the structure. This phenomenon is doubtlessly the most relevant to engineering structures. Indeed, past research on localization in structures has mainly focused on strong localization effects $[2,3,5-8]$, while most work in physics has been concerned with weak localization. This important distinction seems to be unclear in the structural dynamics literature, as it is rarely stated clearly which type of localization-weak or strong-is being studied.

\section{CONCLUSIONS}

The effects of disorder on vibration propagation in chains of coupled oscillators have been studied by a probabilistic approach. The phenomena of weak and strong localization have been shown to occur for strong and weak coupling between oscillators, respectively. Asymptotic expressions have been derived for the localization factors in these two limiting cases and have been confirmed by Monte Carlo simulations. While weak localization phenomena are probably of little concern to the structural dynamicist, small disorder has been shown to cause a drastic spatial attenuation of the vibration amplitude in the weak coupling case.

\section{ACKNOWLEDGMENT}

This work is supported by National Science Foundation Grant No. MSM-8700820, Dynamic Systems and Control Program. Dr Elbert L. Marsh is the grant monitor.

\section{REFERENCES}

1. N. A. VALERO and O. O. BENDIKSEN 1986 American Society of Mechanical Engineers Journal of Engineering for Gas Turbines and Power 108(2), 293-299. Vibration characteristics of mistuned shrouded blade assemblies. 
2. S. T. WEI and C. PIERRE 1988 American Society of Mechanical Engineers Journal of Vibration, Acoustics, Stress, and Reliability in Design 110(4), 429-438. Localization phenomena in mistuned assemblies with cyclic symmetry, Part I: free vibrations.

3. C. Pierre, D. M. TANG and E. H. Dowell 1987 American Institute of Aeronautics and Astronautics Journal 25(9), 1249-1257. Localized vibrations of disordered multi-span beams: theory and experiment.

4. C. H. Hodges and J. Woodhouse 1983 Journal of the Acoustical Society of America 74(3), 894-905. Vibration isolation from irregularity in a nearly periodic structure: theory and measurements.

5. O. O. BENDIKSEN 1987 American Institute of Aeronautics and Astronautics Journal 25(9), 1241-1248. Mode localization phenomena in large space structures.

6. P. J. COR NWELL and O. O. BENDIKSEN 1989 American Institute of Aeronautics and Astronautics Journal 27(2), 219-226. Localization of vibrations in large space reflectors.

7. C. PIERRE and P. D. CHA 1989 American Institute of Aeronautics and Astronautics Journal 27(2), 227 241. Strong mode localization in nearly periodic disordered structures.

8. C. PierRe and E. H. DOWELL 1987 Journal of Sound and Vibration 114, 549-564. Localization of vibrations by structural irregularity.

9. C. H. HodGES 1982 Journal of Sound and Vibration 82, 441-424. Confinement of vibration by structural irregularity.

10. G. J. KISSEL 1987 AIAA Paper 87-0819, Proceedings of the 28th AIAA/ASME/ASCE/AHS Structures, Structural Dynamics and Materials Conference, Monterey, California. Localization in disordered periodic structures.

11. G. J. KISSEL 1988 Ph.D. Dissertation, Massachusetts Institute of Technology. Localization in disordered periodic structures.

12. R. A. IbRAHIM 1987 Applied Mechanics Reviews 40(3), 309-328. Structural dynamics with parameter uncertainties.

13. H. MATSUDA and K. ISHII 1970 Supplement of the Progress of Theoretical Physics 45, 56-86. Localization of normal modes and energy transport in the disordered harmonic chain.

14. D. C. Herbert and R. Jones 1971 Journal of Physics C: Solid State Physics 4, 1145-1161. Localized states in disordered systems.

15. K. ISHII 1973 Supplement of the Progress of Theoretical Physics 53, 77-138. Localization of eigenstates and transport phenomena in the one-dimensional disordered system.

16. P. D. KIRKMAN and J. B. PENDRY 1984 Journal of Physics C: Solid State Physics 17, 4327-4344. The statistics of one-dimensional resistances.

17. J. F. M. SCOTT 1985 Proceedings of the Royal Society of London A398, 341-363. The statistics of waves propagating in a one-dimensional random medium.

18. C. H. Hodges and J. WoOdhouse 1986 Reports in Progress in Physics 49, 107-170. Theories of noise and vibration transmission in complex structures.

19. D. J. MEAD 1975 Journal of Sound and Vibration 40,1-18. Wave propagation and natural modes in periodic systems, I: mono-coupled systems.

20. L. BRILlouiN 1953 Wave Propagation in Periodic Structures. New York: Dover. Second edition.

21. R. C. ENGELS 1980 Journal of Sound and Vibration 69, 181-197. Response of infinite periodic structures.

22. F. Y. CHEN 1971 Journal of Sound and Vibration 14, 57-79. On modeling and direct solution of certain free vibration systems.

23. I. S. GRADSHTEYN and I. M. RYZHIK 1980 Table of Integrals, Series, and Products. Orlando: Academic Press. Corrected and enlarged edition.

24. R. COURANT and D. Hilbert 1953 Methods of Mathematical Physics, Volume I. New York: Interscience. 